\title{
Propositions pour une approche géocritique de la littérature swahilie
}

Xavier Garnier

\section{(2) OpenEdition}

1 Journals

Édition électronique

URL : https://journals.openedition.org/eastafrica/599

DOI : $10.4000 /$ eastafrica.599

ISSN : 2790-1076

\section{Éditeur}

IFRA - Institut Français de Recherche en Afrique

\section{Édition imprimée}

Date de publication : 1 septembre 2008

Pagination : 135-150

ISSN : 2071-7245

\section{Référence électronique}

Xavier Garnier, «Propositions pour une approche géocritique de la littérature swahilie », Les Cahiers d'Afrique de l'Est / The East African Review [En ligne], 40 | 2008, mis en ligne le 07 mai 2019, consulté le 09 décembre 2021. URL : http://journals.openedition.org/eastafrica/599; DOI : https://doi.org/ 10.4000/eastafrica.599

Ce document a été généré automatiquement le 9 décembre 2021.

Les Cahiers d'Afrique de l'Est / The East African Review 


\title{
Propositions pour une approche géocritique de la littérature swahilie
}

\author{
Xavier Garnier
}

1 Les études sur la littérature swahilie ont jusqu'ici essentiellement privilégié les mises en perspectives temporelles, en état attentives aux problèmes de chronologie et de succession de générations. En ce qui concerne la poésie classique, le travail philologique sur les manuscrits anciens, l'importance des questions généalogiques dans le discours des poètes, mais également les lectures socio-historiques qui ont été faites de la littérature moderne et l'importance accordée à la périodisation de cette littérature, toutes ces modalités de l'approche critique s'inscrivent dans un cadre temporel. Nous proposons ici des hypothèses d'ordre méthodologique pour appréhender le corpus littéraire swahili dans sa relation à l'espace.

2 Un tel projet répond à une double ambition, l'une interne aux études littéraires, l'autre externe :

- ouvrir de nouvelles lectures critiques pour le corpus littéraire swahili ;

- faire apparaître le rôle et éventuellement l'impact de la littérature et plus largement des textes dans la définition et la configuration d'un éventuel « espace swahili ».

Ces considérations s'inscrivent dans le cadre plus large d'une réflexion d'ordre «géocritique » sur l'articulation, ou l'interaction entre textes littéraires et espaces géographiques, à partir de la proposition de Bertrand Westphal : «L'espace transposé en littérature influe sur la représentation de l'espace dit réel (référentiel), sur cet espace-souche dont il activera certaines virtualités ignorées jusque-là ou ré-orientera la lecture $»^{1}$. La littérature ne sera pas appréhendée pour sa façon de représenter l'«espace-souche" référentiel, mais pour ses capacités à configurer cet espace en influant sur la façon dont on le fait exister en le percevant. La perception d'un «monde swahili » induit une forme d'espace, c'est précisément au niveau des processus de mise en forme de l'espace que la littérature intervient. 

entre les textes et l'espace :

- on partira de l' "espace-souche», pour analyser l'influence des données de géographie physique et humaine sur les répartitions et les dynamiques littéraires ;

- on s'intéressera ensuite à ce que les textes retiennent des lieux, à la façon dont ils les réagencent dans un lieu propre dès lors qu'ils font «œuvre ";

- on terminera en abordant la question de l'inscription des textes dans l'aire swahilie et de la façon dont celle-ci permet une orientation.

5

On peut noter que les problématiques linguistiques n'apparaissent pas dans cette introduction, non que la langue soit étrangère à ce dont il va être question. Le parti pris méthodologique est de mettre entre parenthèses la langue, comme lien trop évident entre les textes et les territoires, pour faire apparaitre d'autres types de liens, qui ne sont certes pas étrangers à la langue en elle-même, mais peut-être à la façon dont on tend parfois à l'objectiver. On pourrait en dire autant de la notion fort complexe de «culture ». Il faut prendre les propositions méthodologiques qui vont suivre comme des moyens indirects mis en œuvre pour travailler à partir de problématiques nouvelles sur les questions de la langue et de la culture swahilies.

\section{Les lieux et leur potentiel énonciatif}

6 Un premier type d'approche consiste à partir des lieux pour examiner leur capacité à générer des textes. De façon un peu théorique on prendrait le lieu comme instance énonciative, ou tout du moins comme entité susceptible d'influencer les formes. L'existence d'un territoire est nécessaire à l'existence de ce que Deleuze et Guattari appellent un "agencement collectif d'énonciation »: ils prennent l'exemple du café, comme lieu où est susceptible de se forger un type d'énonciation spécifique, produisant des énoncés qui marquent un territoire.

Dans le contexte swahili on pourrait travailler sur différents types de lieux :

\subsection{Les villes}

Les villes sont les entités géographiques qui ont jusqu'ici été le plus souvent prises en considération par les approches " géocritiques » mais, concernant le continent africain, elles restent peu étudiées en France dans cette perspective. La ville est un type d'espace intéressant pour la zone swahilie, précisément parce qu'elle est considérée depuis longtemps comme matricielle pour la littérature. Mombasa, mais aussi Tanga, sont des entités urbaines perçues depuis longtemps comme génératrices de textes, parce qu'elles ont été portées haut par des poètes.

\subsubsection{Ville et vie poétique}

Dans l'enquête très précieuse qui a été menée par T.S.Y. Sengo et M.M. Mulokozi au début des années 1990 sur les poètes swahilis, les villes sont les points de référence de la cartographie des poètes qui est proposée ${ }^{2}$. La récolte des informations a été permise par un véritable travail de terrain, effectué de façon systématique à partir des villes et de leur zone d'influence. Il est intéressant de noter à quel point les noms des poètes semblent étroitement devoir être attachés à des noms de ville, même lorsque ceux-ci 
n'y sont pas restés au cours de leur vie. Cette territorialisation de la poésie mérite qu'on s'y attarde un petit peu. L'association d'un nom de poète à un nom de ville n'est semble-t-il qu'un moyen terme. L'enquête de Sengo et Mulokozi montre que dans de nombreux cas, c'est à un village proche, voire à un quartier précis que le poète est référé3.

10 La lecture du rapport d'enquête de Sengo et Mulokozi fait apparaître de façon claire que la répartition entre poésie urbaine et poésie rurale. Ils attirent l'attention sur le cas particulier de Bukoba, ou les poètes ne vivent pas en ville, mais sont répartis dans les villages environnants, comme c'est le cas sur la côte.

\subsubsection{La ville comme lieu énonciatif}

11 On peut également enquêter sur la vie littéraire d'une ville de façon ouverte : à la fois transgénérique, transculturelle et translinguistique. C'est le travail que fait actuellement Maëline Le Lay, doctorante à l'Université Paris 13, sur Lubumbashi. On pourrait bien sûr lancer de telles enquêtes sur de nombreuses autres villes de l'aire swahili, à commencer par les deux principales: Nairobi et Dar es Salaam, dont la " cartographie littéraire » reste semble-t-il encore à faire.

Outre un indispensable bilan sur les politiques culturelles des municipalités, il est toujours précieux de localiser les éditeurs (parfois très discrets), les scènes théâtrales, les points de vente, les imprimeries, etc. La prise en compte des rumeurs de quartier, et la façon dont elles peuvent éventuellement être relayées par une presse locale ; l'étude du contexte d'émergence de tel ou tel phénomène culturel qui se serait propagé à partir d'un quartier où d'un lieu identifiable.

13 À la fois fascinante et inquiétante la technique du spiritual mapping qui se développe beaucoup dans les milieux religieux, consistant à cartographier les territoires à convertir, avec un fort accent mis sur les villes, perçues comme forteresses à conquérir, avec identification des lieux de résistance, etc.

\subsection{La notion de « lieu-clé »}

Il y a chez le philosophe Gilbert Simondon de précieuses propositions sur le lien entre l'homme et la nature par le biais de la notion de point-clé ou de lieu remarquable :

«Dans la totalité constituée par l'homme et le monde apparaît comme première structure un réseau de points privilégiés réalisant l'insertion de l'effort humain, et à travers lesquels s'effectuent les échanges entre l'homme et le monde. Chaque point singulier concentre en lui la capacité de commander à une partie du monde qu'il représente particulièrement et dont il traduit la réalité, dans la communication avec l'homme. On pourrait nommer ces points singuliers des pointsclés commandant le rapport homme-monde, de manière réversible, car le monde influence l'homme comme l'homme influence le monde. Tels sont le sommet des montagnes ou certains défilés, naturellement magiques, parce qu'ils gouvernent une contrée. Le cœur de la forêt, le centre d'une plaine ne sont pas seulement des réalités géographiques métaphoriquement ou géométriquement désignées : ce sont des réalités qui concentrent les pouvoirs naturels comme elles focalisent l'effort humain : elles sont des structures figurales par rapport à la masse qui les supporte, et qui constitue leur fond. » (Simondon, 1989 : 165). 
15 On pourrait travailler sur ces lieux remarquables et la manière dont ils suscitent une littérature. Le travail de Nathalie Carré, qui commence une thèse de doctorat sur les textes des voyageurs swahili rassemblés par Velten, pourra ouvrir une telle piste.

\subsubsection{Lieux touristiques}

16 Les sites touristiques de l'aire swahilie génèrent une production textuelle dont nous pouvons tenir compte. Il y a, à tout point du territoire, des curiosités, qui sont des points de cristallisation d'une activité touristique plus ou moins développée, et qui sont en même temps des générateurs de discours (légendes, anecdotes, etc.).

17 Les montagnes sont bien sûr de tels points remarquables dont on peut essayer de mesurer l'impact sur la production textuelle.

\subsubsection{La côte comme lieu-clé}

18 La question de civilisation swahilie comme civilisation côtière, qui a fait couler beaucoup d'encre, a jusqu'ici été posée davantage en termes culturels que géographiques. On pourrait voir la côte, non pas simplement dans sa différence à l'intérieur, mais également à l'insulaire. On aurait donc trois termes : kisiwa, mrima et bara, renvoyant à trois types d'espaces que l'on pourrait d'un point de vue de géographie physique.

19 La définition donnée pour mrima dans le Kamusi est « une bande d'une largeur de vingt kilomètres à partir du rivage $»^{4}$, difficile d'imaginer un découpage plus précis et plus culturellement neutre. Le rivage est le point d'intensité de la côte, il en est la ligne remarquable.

On pourrait voir l'île comme une côte qui s'est repliée sur elle-même, encerclée par son rivage. Dans les deux cas, la côte et l'île, l'espace est envisagé à partir d'un point d'intensité, un lieu remarquable, dont il est possible de faire l'expérience : le rivage.

On comprend que la notion d'intérieur est la plus relative, puisqu'elle ne peut être saisie autrement que par abstraction. L'expérience de l'intérieur ne pourra se faire qu'à l'aide d'autres points-clés.

\subsubsection{Les lieux de mémoire}

Identification de lieux porteurs d'une mémoire historique pour la collectivité swahilie. On pense bien sûr aux ruines de Kilwa, ou de Paté. Mais aussi au point de rencontre Stanley/Livingston à Ujiji, etc.

\subsection{Voies de communication}

23 La cohérence de la bande côtière peut également être vue du point de vue des voies de communication, le cabotage assurant un lien entre les différentes villes côtières. On peut en dire autant des fleuves, des routes et des voies ferrées. La notion de désenclavement est bien sûr fondamentale pour la vie littéraire, et on pourrait fort bien imaginer une enquête sur le TAZARA, la ligne Dar es Salaam-Kigoma, ou encore Mombasa-Kampala comme instance énonciative. 
Je reviendrai sur cette question à propos des questions de propagation de la littérature populaire (III.2)

\subsection{Lieux de la diaspora swahili}

La question des productions discursives au sein des diasporas, souvent abordées du point de vue de leur « déterritorialisation » supposée, pourra être renouvelée par notre approche notamment par l'identification de lieux de production discursive diasporiques, comme certains quartiers, les foyers, voire les cafés... ou encore, les «sites" internet comme lieux technologiques dont on étudiera l'influence sur les formes de discours.

\section{Exemples d'enjeux spatiaux au sein des textes swahili}

Gilles Deleuze et Félix Guattari proposent, dans Mille Plateaux, une distinction entre l'espace strié et l'espace lisse qui peut nous servir de guide pour comprendre la nature du travail que la littérature fait sur l'espace (Deleuze et Guattari, 1981: 592-625). L'espace strié est extensif, il met en jeu des structurations, il correspond à un espace disposé à la mesure et au découpage. Les pouvoirs étatiques appréhendent les territoires comme des espaces striés. L'espace lisse est intensif, il échappe à la dimension et à la structure par ses dynamiques directionnelles, il n'est pas peuplé d'individus mais d'événements. L'important pour nous est de comprendre qu'il ne s'agit pas de deux types d'espaces hétérogènes, mais de deux façons de saisir l'espace qui sont étroitement corrélées. La littérature joue de la circulation entre ces deux modalités de l'espace.

27 L'approche géocritique naît de la mise en discussion de la vocation «mimétique » de la littérature. Il n'y a pas de représentation des lieux, mais toujours un «travail» sur les lieux, ou l'espace. Voici une proposition de classification des types d'opération littéraire sur l'espace, par genres...

\subsection{Usage poétique des toponymes : dire l'intensité des lieux}

Je suis frappé par le succès de l'énumération des toponymes, particulièrement des noms de villes, par exemple dans la chanson populaire. Ce phénomène n'est pas spécifique au monde swahili. Décliner les noms de lieux pour faire exister un poétiquement un espace est un moyen efficace pour concentrer dans le langage la charge émotive d'un lieu. Par le nom propre l'espace se transfère directement dans le langage.

Derrière il y a la question de l'ancrage territorial de la langue par la poésie. Dans quelle mesure la poésie swahilie est liée à une territorialisation de la langue et tend-elle établir un lien d'intensité entre une langue et des lieux? 


\subsection{Le théâtre ou la mise en dialectique de l'espace strié (multifocalité)} qui font l'objet d'un consensus social. L'espace est structuré en amont de la fable (romanesque ou théâtrale) et celle-ci s'appuie sur cette structure spatiale pour structurer la fable. Le théâtre swahili a, me semble-t-il, particulièrement posé cette question. Une pièce comme Kwenye ukingo wa Thim d'Ebrahim Hussein, met en scène l'antagonisme entre Luo et Gikuyu au sein du swahili, à partir d'un conflit concernant le lieu de sépulture d'un personnage. D’une façon générale, le théâtre de Hussein fait jouer les différentes logiques de structuration de l'espace.

\subsection{Le roman : vers l'espace lisse}

\subsubsection{Le roman policier ou l'autre regard}

$\mathrm{Du}$ point de vue de la perception de l'espace lisse, il faut noter l'importance du personnage de Bwana Msa dans les romans de Mohammed Abdulla : celui qui regarde à l'envers, pour dissoudre les énigmes. Les énigmes policières sont toujours pour lui un effet de perspective. Bwana Msa ne les résout pas parce qu'il est plus intelligent que les autres, mais parce qu'il est capable de décloisonner les espaces. S'il fallait chercher un modèle à Bwana Msa, se serait davantage chez le Dupin d'Edgar Poe que chez Sherlock Holmes (qu'il revendique pourtant). Les romans de Mohammed Abdulla, sont centrifuges : ils partent d'un cas très localisé, enserré dans un fort marquage culturel et social, et trouvent leur résolution par une ouverture à un espace mondial décloisonné (avec mariages interraciaux, flux d'argent et changements d'identités, etc.)

\subsubsection{Kezilahabi et l'espace polarisé}

On peut proposer une lecture spatiale de l'œuvre romanesque de Kezilahabi à partir du motif du cercle. De Rosa Mistika à Mzingile, le cercle est omniprésent dans les romans et toujours selon un modèle similaire: les personnages font cercle autour d'un point d'incandescence, qui peut être un feu, mais également un danseur. En faisant cercle on se détourne de la structure sociale pour boire et libérer la parole. Le cercle est la configuration spatiale propice au vertige.

On pourrait montrer comment le cercle fonctionne chez Kezilahabi comme un envers de la structure sociale. Le cercle est chez Kezilahabi un trou noir qui fait fuir la société, son entreprise narrative est liée à l'émergence de cet espace. L'œuvre de Kezilahabi serait une réponse narrative à la mise en place d'un quadrillage bureaucratique de l'espace social, tel qu'il s'est mis en place depuis l'époque coloniale. La vertu initiatique, voire mystique du cercle apparaîtra de façon explicite dans Nagona et Mzingile.

\subsubsection{Espaces anomiques ou labyrinthiques}

Au début de la nouvelle de Mohamed Suleiman intitulée «Mji » (Suleiman, 1978) un jeune broussard doit se rendre chez son cousin à Dar es Salaam, muni d'indications pour trouver l'itinéraire, mais il descend du bus une station trop tôt et du coup ne peut plus se repérer. La ville devient pour lui un labyrinthe. Beaucoup de romans qui 
racontent comment un personnage est détourné de sa trajectoire habituelle (on trouvera un bel exemple de cette remontée du labyrinthe au sein d'un environnement quotidien dans Kufa kuzikana de Walibora...).

L'apparition du merveilleux dans le roman actuel peut être comprise comme un déplacement du regard. C'est explicitement le cas dans Babu alipofufuka de Mohamed Said. Tentatives pour voir le monde depuis nulle part, être un pur regard non situé (cf. Dunia yao) : transforme le monde en une pure visibilité...

\section{Quel espace pour la littérature swahilie?}

Il s'agit maintenant d'examiner la façon dont la littérature configure un espace pour sa propre inscription. Poser les questions de réception en termes spatiaux permet d'ouvrir la question de l'espace de vie des textes littéraires. En s'inscrivant dans l'espace, ils orientent leur propre espace de vie, et par là agissent sur le réel.

\subsection{La querelle du vers libre : quel espace pour la poésie ?}

La querelle sur le vers libre qui a divisé la communauté poétique n'était pas simplement un problème de moyens formels pour la poésie (cela a tout de suite été vu), ni même une simple question de rattachement culturel (les poètes de l'intérieur contre les poètes de la côte ou la volonté, de la part des réformateurs, de détacher la poésie swahili du modèle arabe). Il y a également une divergence dans la conception de l'espace de réception pour la poésie. Du point de vue des réformateurs, l'horizon de la poésie serait l'espace strié de la société. Au plus fort de la querelle ils sont appelés " ceux de la colline ", c'est-à-dire les universitaires attachés à leur campus, faisant de la poésie à partir d'une position institutionnelle.

De l'autre côté les «traditionalistes » mettent la poésie en lien avec un espace lisse, beaucoup plus informel. Je pense à cette image de Haji Gora, rapportée par Samson Ridder, du poème comme un bateau. Les différents types de poèmes étant comparables à différents types de bateau, ayant chacun leur particularité de construction, mais avec une exigence commune de bon équilibre pour pouvoir tenir la mer et ne pas couler. Cette image très forte du poème comme un bateau flottant sur un élément liquide, parfois agité, toujours en mouvement me semble très intéressante.

D'un côté une poésie qui rompt avec les contraintes formelles, mais qui peut le faire en s'appuyant explicitement ou implicitement sur une stabilité institutionnelle et la foi en une organisation sociale. Un révélateur de cette tendance est le lien entre la poésie intimiste et le refus des contraintes formelles (cf. «Kariha » dans Jicho la Ndani de Said A. Mohamed. Voir aussi les titres des recueils de Kezilahabi : Karibu Ndani et Kichomi) Cet avènement d'une poésie personnelle n'est-il pas lié à l'existence d'un espace personnel qui est la garantie de sa cohérence?

Du côté des traditionalistes, la poésie se réfère moins à la personne qu'à une collectivité non socialisée. La poésie est adressée à une foule présente et à venir. Un mot-clé de cette poésie serait la gloire (sifa), on ne saurait être poète si l'on ne vise la gloire ! La gloire est à distinguer de la reconnaissance sociale (chère à Bourdieu), la gloire est à considérer comme un flux, elle arrive par vague depuis l'élément liquide qu'est la foule (cf. Shaaban Robert). 
Différence entre une poésie qui s'adresse à des individus et une poésie qui interpelle une foule : deux espaces différents pour la poésie.

\subsection{L'espace proliférant du roman populaire}

La distinction entre un roman savant et un roman populaire dans l'espace swahili a été posée très tôt, surtout à partir de la question du roman policier, considéré au début comme un genre populaire plutôt dommageable. Encore une fois on peut prendre la dualité savant/populaire d'un point de vue spatial. Le roman populaire doit son succès à la façon dont il travaille avec les affects individuels immanents : rêves d'amour et de luxe, mais aussi besoin de sécurité et pulsions de vengeance, goût pour le voyeurisme, etc. Les intellectuels reprochent au roman populaire de flatter les pulsions et d'être idéologiquement conformiste. C'est à ce prix que la littérature populaire pénètre sans difficulté l'espace social, elle est en prise avec les populations... mais cette propagation se fait au risque d'un renoncement à l'ambition littéraire, qui est de créer son espace de création propre et d'y capter des lecteurs.

Pourtant cette vitalité de la littérature populaire, aussi trash soit-elle, est peut-être une chance pour la littérature swahili, par ce lien qu'elle établit avec le territoire. Via la presse, mais aussi les petits romans brochés, la fiction narrative en prose est présente presque partout. Il serait intéressant de suivre les modalités de diffusion de cette littérature populaire et s'il y a des effets localisation pour certains auteurs.

La particularité de la littérature savante, au moins jusqu'à la fin des années 1980 en Tanzanie, était de se servir du roman pour aborder des questions sociétales. En ce sens le roman adopte un point de vue surplombant. Les personnages et les histoires servent à illustrer une démonstration ou une dénonciation. Tendance à une littérature " mot d'ordre ", adossée à une volonté politique de transformation sociale. C'est le moment de l'antagonisme maximum avec la littérature populaire, considérée comme «irresponsable». Du coup l'espace naturel de cette littérature savante a été l'institution scolaire, qui a permis une diffusion systématique de certains textes "classiques", mais sur un mode totalement différent : non par propagation mais par répartition. Il est intéressant de constater que l'institution scolaire kényane, n'a eu aucun problème à intégrer les classiques tanzaniens, alors que la littérature populaire ne passe pas les frontières (à lier à la territorialisation de la littérature populaire).

Il me semble qu'un point de rencontre significatif entre littérature savante et littérature populaire est lié à la plus grande prise en compte des espaces territoriaux (cf. Makuadi wa soko huru de Chachage et son attention au delta de la Rufiji ; cf. la précision des références aux lieux chez Ben Mtobwa.)

Cela ne contredit pas l'abandon du réalisme par le roman contemporain, au contraire. La tendance actuelle au réalisme- merveilleux dans la littérature d'Afrique de l'Est est à mon avis moins l'effet d'une déterritorialisation au nom de la globalisation, que le signe d'un effort pour ancrer cette littérature dans des lieux concrets, qui ne seraient plus des points surplombants d'où s'énonce une parole totale, mais des points locaux à partir desquels se configure un espace. 


\section{BIBLIOGRAPHIE}

Chachage, C.S.L. (2002). Makuadi wa soko huria, Dar es Salaam : E\&D.

Deleuze, Gilles et Guattari, Félix (1981). Mille Plateaux, Paris : Minuit.

Garnier, Xavier et Zoberman, Pierre (eds.) (2006). Qu'est-ce qu'un espace littéraire ? Paris : Presses universitaires de Vincennes.

Hussein, Ebrahim (1976). Kwenye ukingo wa Thym, Nairobi : Oxford University Press.

Kezilahabi, Euphrase (1990). Nagona, Dar es Salaam, Dar es Salaam University Press.

Kezilahabi, Euphrase (1991), Mzingile, Dar es Salaam : Dar es Salaam University Press.

Sengo T.S.Y. \& Mulokozi, M.M. (1994). Research on the history of Kiswahili poetry. A.D. 1000-2000, Final Report, OSSREA/IKR, University of Dar es Salaam.

Simondon, Gilbert (1989 [1958]). Du mode d'existence des objets techniques. Paris : Aubier.

Suleiman, Mohamed (1978). Kicheko cha ushindi. Nairobi : Shungwaya Publishers.

Westphal, Bertrand (2007). La géocritique. Réel, fiction, espace, Paris : Minuit.

Walibora, Ken (2003). Kufa kuzikana, Nairobi : Longhorn Publishers.

\section{NOTES}

1. Westphal (2000) : 21 .

2. Sengo \& Mulokozi (1994).

3. Je suis par exemple curieux d'en savoir davantage sur Mnyanjani, près de Tanga, village auquel sont référés un grand nombre de poètes (Gulam Mabondo bin Mwinyimatano, Mdanzi Hanasa, etc.)

4. «Ukanda wa pwani kiasi cha kilometa ishirini kwendea bara. »

\section{AUTEUR}

\section{XAVIER GARNIER}

Cenel - Université Paris 13 